Pacific Journal of Mathematics

SUMMABILITY OF MATRIX TRANSFORMS OF
STRETCHING AND SUBSEQUENCES 


\title{
SUMMABILITY OF MATRIX TRANSFORMS OF STRETCHINGS AND SUBSEQUENCES
}

\author{
DAVID F. DAWSON
}

It is well known that if a regular matrix sums every subsequence of a sequence $x$, then $x$ converges. It follows trivially from this result and row finiteness of the Cesaro summability matrix that if $A$ is a regular matrix such that $A y$ is Cesaro summable for every subsequence $y$ of $x$, then $x$ is convergent (not merely Cesaro summable). The purpose of the present paper is to give some general results of this type involving matrix methods that are not necessarily row finite. For example, it is shown that if $T$ is any regular matrix summability method and $A$ is a regular matrix such that $A y$ is absolutely $T$-summable for every stretching $y$ of $x$, then $x$ is absolutely convergent. This is done without assuming that $x$ is bounded, and consequently, without the benefit of associativity.

The well known result mentioned above is due to R. C. Buck [2], and the trivial consequence involving the Cesàro summability matrix $(C, 1)$ can be seen as follows. If $A$ is regular and $A y$ is Cesàro summable for every subsequence $y$ of $x$, then $(C, 1) A$ is a regular matrix which sums every subsequence of $x$, since row finiteness of $(C, 1)$ gives the associativity relation $(C, 1)(A y)=[(C, 1) A] y$. Consequently by Buck's theorem, $x$ is convergent.

When we say that a matrix $A$ is semiregular, we will mean that $A$ is regular over the set of all convergent sequences of 0 's and 1's. Thus $A=\left(a_{p q}\right)$ is semiregular iff $A$ satisfies the first two of the following three conditions for regularity:

1) $a_{p q} \rightarrow 0$ as $p \rightarrow \infty, q=1,2,3, \cdots$,

2) $\sum_{q=1}^{\infty} a_{p q} \rightarrow 1$ as $p \rightarrow \infty$,

3) $\sum_{q=1}^{\infty}\left|a_{p q}\right|<K_{A}, \quad p=1,2,3, \cdots$.

If $\varepsilon$ is a positive term null sequence and each of $x$ and $y$ is a complex sequence, then the statement that $y$ contains an $\varepsilon$-copy of $x$ means that $y$ contains a subsequence $\left\{y_{n_{p}}\right\}$ such that $\left|y_{n_{p}}-x_{p}\right|<$ $\varepsilon_{p}, p=1,2,3, \cdots$.

THEOREM 1. If $T=\left(t_{p q}\right)$ is a matrix such that $\sum_{q=1}^{\infty}\left|t_{p q}\right|<L_{p}$, $p=1,2,3, \cdots, A$ is a regular matrix, and $A y$ is T-summable for every subsequence $y$ of $x$, then either $x$ converges or TA is a Schur matrix, i.e., TA sums every bounded sequence.

Proof. Suppose $x$ is unbounded. Clearly $A$ is row finite since 
$A z$ is defined for every subsequence $z$ of $x$. It is somewhat less clear, but nonetheless true, that $T$ is row finite, and we gives a proof. Suppose the $p$ th row of $T$ contains infinitely many nonzero terms. Using only the semiregularity of $A$, we can construct a subsequence $y$ of $x$ such that $\left|t_{p j} \sum_{q=1}^{\infty} a_{j q} y_{q}\right|=\left|t_{p j}(A y)_{j}\right|>1$ for infinitely many values of $j$, thus ruling out convergence of $\sum_{q=1}^{\infty} t_{p q}(A y)_{q}$, and contradicting the fact that $T(A y)$ is defined. We see this as follows. Suppose a finite subsequence $y_{1}, y_{2}, \cdots, y_{n}$ of $x$ has been determined. From the semiregularity of $A$, there exists a positive integer $v$ such that if $i>v$, then $\sum_{q=1}^{n}\left|a_{i q}\right|<1 / 2$ and $\left|\sum_{q=1}^{\infty} a_{i q}-1\right|<1 / 2$. Choose $j>v$ such that $t_{p j} \neq 0$. Let $a_{j r}$ be the last nonzero term in the $j$ th row of $A$. Then from the inequalities above, $r>n$. Determine $y_{n+1}, \cdots, y_{r}$ such that $y_{1}, \cdots, y_{r}$ is a finite subsequence of $x$ and

$$
\left|a_{j r} y_{r}\right|>\frac{1}{\left|t_{p j}\right|}+\left|\sum_{q=1}^{r-1} a_{j q} y_{q}\right|
$$

Then regardless of how the remaining terms of $y$ are chosen,

$$
\left|(A y)_{j}\right|=\left|\sum_{q=1}^{\infty} a_{j q} y_{q}\right|=\left|\sum_{q=1}^{r} a_{j q} y_{q}\right|>\frac{1}{\left|t_{p j}\right|},
$$

thus establishing our assertion above. Therefore $T$ must be row finite. Hence the associativity $T(A z)=(T A) z$ holds for all $z$. Therefore $T A$ sums every subsequence of the divergent sequence $x$. Thus by the theorem in [9], $T A$ is a Schur matrix. This completes the proof for the case that $x$ is unbounded.

Next suppose $\left|x_{p}\right|<M, p=1,2,3, \cdots$. We note that if $y$ is any subsequence of $x$, then $\left|\sum_{q=1}^{\infty} t_{p q} \sum_{s=1}^{\infty} a_{q s} y_{s}\right|<M K_{A} \sum_{q=1}^{\infty}\left|t_{p q}\right|<$ $M K_{A} L_{p}$. Thus we can interchange the order of summation and obtain

$$
\sum_{q=1}^{\infty} t_{p q}\left(\sum_{s=1}^{\infty} a_{q s} y_{s}\right)=\sum_{s=1}^{\infty}\left(\sum_{q=1}^{\infty} t_{p q} a_{q s}\right) y_{s}
$$

The left side of $\left(^{*}\right)$ is the $p$ th term of the sequence $T(A y)$ and the right side of $\left(^{*}\right)$ is the $p$ th term of the sequence $(T A) y$. Thus again we have the associativity $T(A y)=(T A) y$. Hence the matrix $T A$ sums every subspace $y$ of $x$. Therefore if $x$ is not convergent, then $T A$ is a Schur matrix by the theorem in [9]. This completes the proof.

THEOREM 2. Suppose $T$ is any regular matrix summability method. If $A$ is a regular matrix such that Ay is T-summable for every subsequence $y$ of $x$, then $x$ is convergent. 
Proof. Since the hypothesis of Theorem 1 is satisfied, then either $x$ converges or $T A$ is a Schur matrix. But $T A$ is regular since it is the product of regular matrices, and no regular matrix is a Schur matrix. This completes the proof.

For stretchings, we obtain the following theorem which is analogous to (but more comprehensive than) Theorem 2.

THEOREM 3. Suppose $T$ is any regular matrix summability method. If $A$ is a regular matrix such that $A y$ is T-summable (absolutely T-summable) for every stretching $y$ of $x$, then $x$ is convergent (absolutely convergent).

We note that Theorem 3 is an immediate consequence of the following result which we shall call the "Copy Theorem."

THEOREM 4. If each of $T$ and $A$ is a regular matrix, $x$ is any complex sequence (bounded or not), and $\varepsilon$ is any positive term null sequence, then there exists a stretching $y$ of $x$ such that $T(A y)$ exists and contains an e-copy of $x$.

Proof. Let $K=K_{A}+K_{T}+\max \varepsilon_{p}+1, M_{p}=1+\sum_{q=1}^{p}\left|x_{q}\right|, \delta_{p}=$ $\min \left\{\varepsilon_{1}, \cdots, \varepsilon_{p}\right\}$, and $Q_{p}=K M_{p}+1$. There exists a positive integer $n_{1}$ such that if $p \geqq n_{1}$, then

$$
\left|\sum_{q=1}^{\infty} a_{p q}-1\right|<\frac{\delta_{1}}{16 Q_{1}}
$$

There exists $r_{1}$ such that

$$
\sum_{q=1}^{n_{1}}\left|t_{r_{1} q}\right|<\frac{\delta_{1}}{8 Q_{1}} \text { and }\left|\sum_{q=n_{1}}^{\infty} t_{r_{1} q}-1\right|<\frac{\delta_{1}}{8 Q_{1}} .
$$

There exists $m_{1}>n_{1}$ such that if $1 \leqq p \leqq r_{1}$ then

$$
\sum_{q=m_{1}}^{\infty}\left|t_{p q}\right|<\frac{\delta_{1}}{8 Q_{2}}
$$

There exists an integer $s_{1}>1$ such that if $1 \leqq p \leqq m_{1}$, then

$$
\sum_{q=s_{1}}^{\infty}\left|a_{p q}\right|<\frac{\delta_{1}}{16 Q_{2}}
$$

Suppose the finite increasing sequences $\left\{n_{p}\right\}_{p=1}^{\alpha-1},\left\{r_{p}\right\}_{p=1}^{\alpha-1},\left\{m_{p}\right\}_{p=1}^{\alpha-1}$, and $\left\{s_{p}\right\}_{p=1}^{\alpha-1}$ of positive integers have been determined. Choose $n_{\alpha}>$ $m_{\alpha-1}$ such that if $p \geqq n_{\alpha}$, then

$$
\left|\sum_{q=s_{\alpha-1}}^{\infty} a_{p q}-1\right|<\frac{\delta_{\alpha}}{16 Q_{\alpha}}
$$


and

$$
\sum_{q=1}^{s_{\alpha}-1}\left|a_{p q}\right|<\frac{\delta_{\alpha}}{8 Q_{\alpha-1}}, \quad \text { where } s_{0}=1 \text { and } M_{0}=1
$$

Choose $r_{\alpha}>r_{\alpha-1}$ such that

$$
\sum_{q=1}^{n_{\alpha}}\left|t_{r_{\alpha} q}\right|<\frac{\delta_{\alpha}}{8 Q_{\alpha}} \text { and }\left|\sum_{q=n_{\alpha}}^{\infty} t_{r_{\alpha} q}-1\right|<\frac{\delta_{\alpha}}{8 Q_{\alpha}} .
$$

Choose $m_{\alpha}>n_{\alpha}$ such that if $1 \leqq p \leqq r_{\alpha}$, then

$$
\sum_{q=m_{\alpha}}^{\infty}\left|t_{p q}\right|<\frac{\delta_{\alpha}}{2^{2+\alpha} Q_{\alpha+1}} .
$$

Choose $s_{\alpha}>s_{\alpha-1}$ such that if $1 \leqq p \leqq m_{\alpha}$, then

$$
\sum_{q=s_{\alpha}}^{\infty}\left|a_{p q}\right|<\frac{\delta_{\alpha}}{2^{3+\alpha} Q_{\alpha+1}} .
$$

From (3) and (4) we can obtain

$$
\left|\sum_{q=n_{\alpha}}^{m_{\alpha}} t_{r_{\alpha} q}-1\right|<\frac{\delta_{\alpha}}{4 Q_{\alpha}} .
$$

From (1) and (5) we obtain

$$
\left|\sum_{q=s_{\alpha-1}}^{s_{\alpha}-1} a_{p q}-1\right|<\frac{\delta_{\alpha}}{8 Q_{\alpha}} \quad \text { for } \quad n_{\alpha} \leqq p \leqq m_{\alpha} .
$$

Thus we have defined the increasing sequences $\left\{n_{p}\right\}_{p=1}^{\infty},\left\{r_{p}\right\}_{p=0}^{\infty},\left\{m_{p}\right\}_{p=0}^{\infty}$, and $\left\{s_{p}\right\}_{p=0}^{\infty}$ of integers, where $r_{0}=0$ and $m_{0}=0$.

Let $\left\{y_{p}\right\}_{p=1}^{\infty}$ be the stretching of $x$ induced by $\left\{s_{p}\right\}_{p=0}^{\infty}[3, \mathrm{p} .455]$. If $\alpha>1$ and $n_{\alpha} \leqq p \leqq m_{\alpha}$, then from (2), (5), and (7) we obtain

$$
\begin{aligned}
\left|\sum_{q=1}^{\infty} a_{p q} y_{q}-x_{\alpha}\right| \leqq & \left|\sum_{q=1}^{s_{\alpha}-1} a_{p q} y_{q}\right|+\left|\sum_{q=s_{\alpha-1}}^{s_{\alpha}-1} a_{p q} y_{q}-x_{\alpha}\right| \\
& +\sum_{v=\alpha+1}^{\infty}\left|\sum_{q=s_{v-1}}^{s_{v}-1} a_{p q} y_{q}\right| \\
\leqq & \left(\sum_{q=1}^{s_{\alpha}-1-1}\left|a_{p q}\right|\right) \max \left\{\left|x_{1}\right|, \cdots,\left|x_{\alpha-1}\right|\right\} \\
& +\left|x_{\alpha}\right|\left|\sum_{q=s_{\alpha}-1}^{s_{\alpha}-1} a_{p q}-1\right|+\sum_{v=\alpha+1}^{\infty}\left|x_{v}\right| \sum_{q=s_{v-1}}^{s_{v}-1}\left|a_{p q}\right| \\
< & \delta_{\alpha} / 8 K+\delta_{\alpha} / 8 K+\sum_{v=\alpha+1}^{\infty}\left|x_{v}\right| \frac{\delta_{v-1}}{2^{2+v} Q_{v}} \\
< & 3 \delta_{\alpha} / 8 K .
\end{aligned}
$$

Also we can prove this inequality for $n_{1} \leqq p \leqq m_{1}$. Thus we have for $\alpha \geqq 1$ and $n_{\alpha} \leqq p \leqq m_{\alpha}$, 
(9)

$$
\sum_{q=1}^{\infty} a_{p q} y_{q}=x_{\alpha}+\mu_{\alpha}, \text { where }\left|\mu_{\alpha}\right|<3 \delta_{\alpha} / 8 K .
$$

If $m_{\alpha-1}<p<n_{\alpha}$, then from (5) we obtain

$$
\begin{aligned}
\left|\sum_{q=1}^{\infty} a_{p q} y_{q}\right| & \leqq\left|\sum_{q=1}^{s_{\alpha}-1} a_{p q} y_{q}\right|+\sum_{v=\alpha}^{\infty}\left|\sum_{q=s_{v}}^{s_{v+1}-1} a_{p q} y_{q}\right| \\
& \leqq\left(\sum_{q=1}^{s_{\alpha}-1}\left|a_{p q}\right|\right) \max \left\{\left|x_{1}\right|, \cdots,\left|x_{\alpha}\right|\right\}+\sum_{q=\alpha}^{\infty}\left|x_{v+1}\right| \sum_{q=s_{v}}^{s_{v+1}-1}\left|a_{p q}\right| \\
& <K M_{\alpha}+\sum_{v=\alpha}^{\infty}\left|x_{v+1}\right| \frac{\delta_{v}}{2^{3+v} Q_{v+1}} \\
& \leqq K M_{\alpha}+\left(\delta_{\alpha} / K\right) \sum_{v=\alpha}^{\infty} 1 / 2^{3+v} \\
& <K M_{\alpha}+1=Q_{\alpha} .
\end{aligned}
$$

From this inequality and (8) we can show that if $m_{\alpha-1}<p \leqq m_{\alpha}$, then

$$
\left|\sum_{q=1}^{\infty} a_{p q} y_{q}\right| \leqq\left|\sum_{q=1}^{\infty} a_{p q}-x_{\alpha}\right|+\left|x_{\alpha}\right|<K M_{\alpha}+1=Q_{\alpha},
$$

since $\left|x_{\alpha}\right|<K M_{\alpha}$ and $3 \delta_{\alpha} / 8 K<1$.

If $r_{i-1}<p \leqq r_{i}$, then from (4) and (10) we obtain

$$
\begin{aligned}
\left|\sum_{q=m_{i}+1}^{\infty} t_{p q}(A y)_{q}\right| & \leqq \sum_{\alpha=i}^{\infty} \sum_{j=m_{\alpha}+1}^{m_{\alpha+1}}\left|t_{p j}\right|\left|(A y)_{j}\right| \\
& \leqq \sum_{\alpha=i}^{\infty} Q_{\alpha+1} \sum_{j=m_{\alpha}+1}^{m_{\alpha+1}}\left|t_{p j}\right| \\
& \leqq \sum_{\alpha=i}^{\infty} Q_{\alpha+1} \frac{\delta_{\alpha}}{2^{2+\alpha} Q_{\alpha+1}}<\delta_{i} / 4 .
\end{aligned}
$$

Thus we see that $T(A y)$ is defined.

From (3), (6), (9), (10), and (11), we obtain

$$
\begin{aligned}
\left|\sum_{j=1}^{\infty} t_{r_{i} j}(A y)_{j}-x_{i}\right| \leqq & \left|\sum_{j=1}^{n_{i}-1} t_{r_{i} j}(A y)_{j}\right|+\left|\sum_{j=n_{i}}^{m_{i}} t_{r_{i} j}(A y)_{j}-x_{i}\right| \\
& +\left|\sum_{j=m_{i}+1}^{\infty} t_{r_{i} j}(A y)_{j}\right| \\
\leqq & Q_{i} \sum_{j=1}^{n_{i}-1}\left|t_{r_{i} j}\right|+\left|\sum_{j=n_{i}}^{m_{i}} t_{r_{i} j}\left(x_{i}+\mu_{i}\right)-x_{i}\right| \\
& +\sum_{\alpha=i}^{\infty}\left|\sum_{j=m_{\alpha^{+}+1}}^{m_{\alpha+1}} t_{r_{i} j}(A y)_{j}\right| \\
< & \delta_{i} / 8+\left|x_{i}\right|\left|\sum_{j=n_{i}}^{m_{i}} t_{r_{i} j}-1\right|+\sum_{j=n_{i}}^{m_{i}}\left|t_{r_{i} j} \mu_{i}\right| \\
& +\sum_{\alpha=i}^{\infty} Q_{\alpha+1} \sum_{j=m_{\alpha}+1}^{m_{\alpha+1}}\left|t_{r_{i} j}\right| \\
< & \delta_{i} / 8+\delta_{i} / 4+3 \delta_{i} / 8+\sum_{\alpha=i}^{\infty} Q_{\alpha+1} \frac{\delta_{\alpha}}{2^{2+\alpha} Q_{\alpha+1}}
\end{aligned}
$$




$$
\begin{aligned}
& <\delta_{i} / 8+\delta_{i} / 4+3 \delta_{i} / 8+\delta_{i} / 4 \\
& =\delta_{i} \\
& \leqq \varepsilon_{i} .
\end{aligned}
$$

This completes the proof.

We can use Theorem 4 to prove the following extension of a theorem of Agnew [1].

THEOREM 5. Suppose $T$ is any regular matrix summability method. If $A$ is a regular matrix and $x$ is a sequence having a finite limit point, then there exists a subsequence $y$ of $x$ such that every finite limit point of $x$ is a T-limit point of $A y$.

Proof. Using the separability of the complex plane, we write the finite limit points of $x$ in a sequence denoted by $u$. Let $v$ denote the sequence $u_{1} ; u_{1}, u_{2} ; u_{1}, u_{2}, u_{3} ; \cdots$, and let $\varepsilon$ be a positive term null sequence. By the "Copy Theorem," there exists a stretching $z$ of $v$ such that $T(A z)$ is defined and contains an $\varepsilon$-copy of $v$. Let $y$ be a subsequence of $x$ such that $z-y$ is a null suquence. Since $T(A y)=T(A[y-z])+T(A z)$, we see that $T(A y)$ is the sum of a null sequence and a sequence which contains an $\varepsilon$-copy of $v$. Therefore every finite limit point of $x$ is a limit point of $T(A y)$. This completes the proof.

In [5] we proved theorems analogous to the results of this paper, except that $T$ was the identity matrix (ordinary convergence) and $A$ was a semiregular matrix. The following theorems are trivial consequences of associativity, the results in [5], and the fact that if $T$ is a row finite regular matrix and $A$ is a semiregular matrix, then $T A$ is a semiregular matrix.

THeOREM 6. Suppose $T$ is any row finite regular matrix summability method. If $A$ is a semiregular matrix such that $A y$ is T-summable for every subsequence $y$ of $x$, then $x$ is convergent.

THEOREM 7. Suppose $T$ is any row finite regular matrix summability method. If $A$ is a semiregular matrix such that $A y$ is T-summable (absolutely T-summable) for every stretching $y$ of $x$, then $x$ is convergent (absolutely convergent).

REMARK. We give an example to show the necessity of "row finite" in the statement "... the fact that if $T$ is a row finite regular matrix and $A$ is a semiregular matrix, then $T A$ is a semiregular matrix," which precedes Theorem 6 . Let $B$ and $A$ be matrices defined as follows: $b_{p q}=2^{p-q-1}$ if $p$ is even and $q \geqq p$, 
$b_{p q}=0$ if $p$ is even and $q<p, b_{p q}=1$ if $p$ is odd and $q=p, b_{p q}=0$ if $p$ is odd and $q \neq p, a_{p q}=0$ if $q<2 p-1$ or $q>2 p, a_{p q}=2^{p-1}+1$ if $q=2 p-1, a_{p q}=-2^{p-1}$ if $q=2 p$. Simple calculations show that $B$ is regular, $A$ is semiregular, but $B A$ is not semiregular.

\section{REFERENCES}

1. R. P. Agnew, Summability of subsequences, Bull. Amer. Math. Soc., 50 (1944), 596-598.

2. R. C. Buck, A note on subsequences, Bull. Amer. Math. Soc., 49 (1943), 898-899.

3. — An addendum to " $A$ note on subsequences," Proc. Amer. Math. Soc., 7 (1956), 1074-1075.

4. D. F. Dawson, Summability of subsequences and other regular transformations of a sequence, Boll. Unione Mat. Ital. (4), 8 (1973), 449-455.

5. - Summability of subsequences and stretchings of sequences, Pacific J. Math., 44 (1973), 455-460.

6. Robert DeVos, Subsequences and rearrangements of sequences in $F K$ spaces, Pacific J. Math., 64 (1976), 129-135.

7. J. A. Fridy, Summability of rearrangements of sequences, Math. Z., 174 (1975), 187-192.

8. Thomas A. Keagy, Matrix transformations and absolute summability, Pacific J. Math., 63 (1976), 411-415.

9. I. J. Maddox, $A$ Tauberian theorem for subsequences, Bull. London Math. Soc., 2 (1970), 63-65.

Received July 13, 1977.

North Texas State University

DENTON, TX 76203 



\section{PACIFIC JOURNAL OF MATHEMATICS}

\section{EDITORS}

RICHARD ARENS (Managing Editor)

University of California

Los Angeles, California 90024

C. W. Curtis

University of Oregon

Eugene, OR 97403

C. C. MOORE

University of California

Berkeley, CA 94720

\section{J. DUGUNDJI}

Department of Mathematics University of Southern California Los Angeles, California 90007

R. Finn AND J. Milgram Stanford University Stanford, California 94305

\section{ASSOCIATE EDITORS}

E. F. BeCK ENBACH

B. H. NeUMaNN

F. WOLF

K. Yoshida

\section{SUPPORTING INSTITUTIONS}

UNIVERSITY OF BRITISH COLUMBIA CALIFORNIA INSTITUTE OF TECHNOLOGY UNIVERSITY OF CALIFORNIA MONTANA STATE UNIVERSITY UNIVERSITY OF NEVADA, RENO NEW MEXICO STATE UNIVERSITY OREGON STATE UNIVERSITY UNIVERSITY OF OREGON
UNIVERSITY OF SOUTHERN CALIFORNIA STANFORD UNIVERSITY UNIVERSITY OF HAWAII UNIVERSITY OF TOKYO UNIVERSITY OF UTAH WASHINGTON STATE UNIVERSITY UNIVERSITY OF WASHINGTON 


\section{Pacific Journal of Mathematics \\ Vol. 77, No. $1 \quad$ January, 1978}

Dan Amir, Chebyshev centers and uniform convexity ............... 1

Lawrence Wasson Baggett, Representations of the Mautner group. I ..... 7

George Benke, Trigonometric approximation theory in compact totally

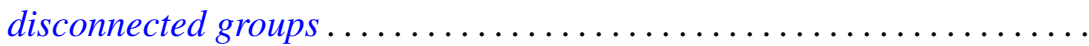

M. Bianchini, O. W. Paques and M. C. Zaine, On the strong compact-ported topology for spaces of holomorphic mappings ..................

Marilyn Breen, Sets with $(d-2)$-dimensional kernels

J. L. Brenner and Allen Kenneth Charnow, Free semigroups of $2 \times 2$

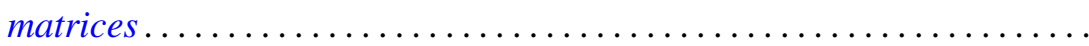

David Bressoud, A new family of partition identities .................

David Fleming Dawson, Summability of matrix transforms of stretchings

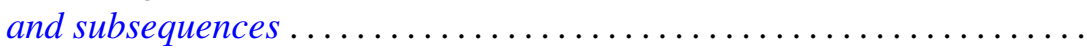

Harold George Diamond and Paul Erdôs, A measure of the nonmonotonicity

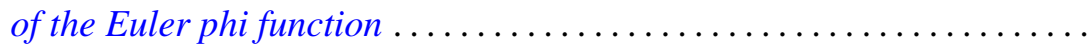

Gary Doyle Faulkner and Ronald Wesley Shonkwiler, Kernel dilation in reproducing kernel Hilbert space and its application to moment

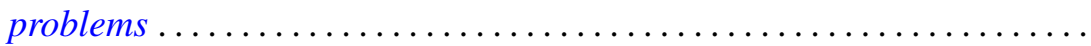

Jan Maksymilian Gronski, Classification of closed sets of attainability in the

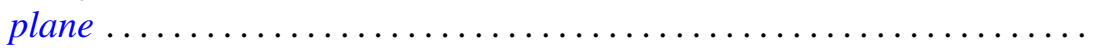

H. B. Hamilton and T. E. Nordahl, Semigroups whose lattice of congruences

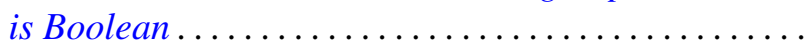

Harvey Bayard Keynes and D. Newton, Minimal $(G, \tau)$-extensions ...

Anthony To-Ming Lau, The Fourier-Stieltjes algebra of a topological

semigroup with involution.

B. C. Oltikar and Luis Ribes, On prosupersolvable groups ...

Brian Lee Peterson, Extensions of pro-affine algebraic groups ...

Thomas M. Phillips, Primitive extensions of Aronszajn spaces ...

Mehdi Radjabalipour, Equivalence of decomposable and 2-decomposable operators. .

M. Satyanarayana, Naturally totally ordered semigroups .

Fred Rex Sinal, A homeomorphism classification of wildly imbedded two-spheres in $S^{3}$

Hugh C. Williams, Some properties of a special set of recurring

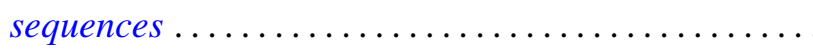

